\title{
Functional CLOCK Is Not Essentially Associated with Metabolic Disruption Caused by Sleep Phase Feeding in Mice
}

\author{
Katsutaka Oishi*,a,b,c,d and Hiroki Okauchi ${ }^{a, b}$ \\ ${ }^{a}$ Biological Clock Research Group, Biomedical Research Institute, National Institute of Advanced Industrial \\ Science and Technology (AIST); Tsukuba, Ibaraki 305-8566, Japan: ${ }^{b}$ Department of Applied Biological Science, \\ Graduate School of Science and Technology, Tokyo University of Science; Noda, Chiba 278-0022, Japan: \\ ${ }^{c}$ Department of Computational Biology and Medical Sciences, Graduate School of Frontier Sciences, The University \\ of Tokyo; Kashiwa, Chiba 277-0882, Japan: and ${ }^{d}$ School of Integrative and Global Majors (SIGMA), University of \\ Tsukuba; Tsukuba, Ibaraki 305-3577, Japan.
}

Received January 8, 2019; accepted March 8, 2019

Consuming food at uncommon times during the day might be associated with obesity in experimental animals and humans. We previously reported that mice become obese and their metabolism becomes disrupted when they consume food during the daytime (sleep phase feeding; SPF), but not during the nighttime (active phase feeding; APF). The goal of the present study was to clarify whether the molecular circadian clock is associated with the mechanisms that underly the metabolic disorders in mice brought about by SPF. We compared the effects of dominant negative Clock gene mutation on metabolic disruption and obesity brought about by SPF in mice. The consumption of food during SP increased body weight, adipose tissue mass and lipogenic gene expression in metabolic tissues, as well as hyperinsulinemia, hyperleptinemia and hepatic lipid accumulation in wild-type and Clock mutant mice, and there were no significant differences between genotypes except for the body weight increase which was attenuated by the Clock mutation. Temporal expression of Per2 was synchronized to feeding rhythms in the liver of both genotypes, although the expression of $D b p$, a representative clock-controlled gene, was significantly damped in peripheral tissues of Clock mutant mice. These findings suggest that the molecular clock is not essentially associated with metabolic disruption caused by SPF. Desynchronized food consumption and central clock-dependent behaviour as well as rhythmic metabolic mechanisms might be associated with the metabolic disruption caused by SPF.

Key words time-restricted food consumption; circadian clock gene; obesity; adiposity; lipogenesis

\section{INTRODUCTION}

The circadian clock controls diurnal variations in behavioral and physiological functions such as movement, sleep-wake regulation, thermogenesis, blood pressure, lipid synthesis and metabolism, as well as hormone secretion. ${ }^{1,2)}$ The core clock genes Clock, Bmall, Per1, Per2, Cryl and Cry2 drive the master clock in the mammalian hypothalamic suprachiasmatic nucleus (SCN) based on transcriptional feedback loops. ${ }^{3)}$ Clock was the first clock gene in vertebrates to be identified in a behavioral screening by forward mutagenesis using $N$-ethyl- $N$ nitrosourea. ${ }^{4)}$ This gene encodes a basic helix-loop-helix-PerArnt-Sim (PAS) transcription factor that positively regulates a feedback loop. Exon 19 in CLOCK is deleted in Clock mutant mice. ${ }^{5)}$ Most peripheral organs such as the heart, lungs, liver, skeletal muscle and adipose tissues that harbor core clock components contain circadian oscillators (peripheral clocks). ${ }^{3)}$ These peripheral clocks are synchronized to the SCN by systemic time cues that include neuronal signals and circulating humoral factors such as glucocorticoids and insulin. ${ }^{1,2)}$ Daily time restricted food consumption evokes rhythmic food anticipation defined as locomotor activity that becomes enhanced two to three hours before food is presented and also independently synchronizes peripheral clocks to the central clock. ${ }^{6,7)}$

Epidemiological studies have suggested that inappropriate habits such as skipping breakfast or consuming food late at night are associated with risk for obesity and disrupted metabolism. ${ }^{8)}$ Time-imposed restricted food consumption affects body weight gain, adiposity and other metabolic parameters in experimental animals. ${ }^{9)}$ We recently showed that mice gained more weight and fat mass, and became hyperinsulinemic, hyperleptinemic and accumulated hepatic lipid with elevated lipogenic gene expression when given access to a high-fat high-sucrose diet during the daytime (sleep phase feeding; SPF) compared with the nighttime (active phase feeding; APF). ${ }^{10-12)}$ The present study aimed to clarify whether CLOCK is associated with obesity and metabolic disruption induced in mice by time-restricted food consumption during the daytime sleep phase.

\section{MATERIALS AND METHODS}

Animals and Study Design Male wild-type and homozygous Clock mutant mouse littermates with an ICR background $^{13,14)}$ were individually housed in cages with running-wheels and given access to normal chow (CE-2; Clea Japan Inc., Tokyo, Japan) ad libitum under a 12-h light-dark cycle (LD 12:12; lights on at Zeitgeber time (ZT) 0 and lights off at ZT12). A white fluorescent lamp provided 330 lx at cage level during the daytime. Amounts of time spent running on wheels recorded throughout the study at 1-min intervals in 1-h bins using Chronobiology Kits (Stanford Software Systems, Stanford, CA, U.S.A.) and averaged during the last $3 \mathrm{~d}$ of food restriction. Twelve-week-old mice were given 2 weeks of access ad libitum to a high-fat high-sucrose diet (F2HFHSD; Oriental Yeast, Tokyo, Japan) comprising 29.1\% 


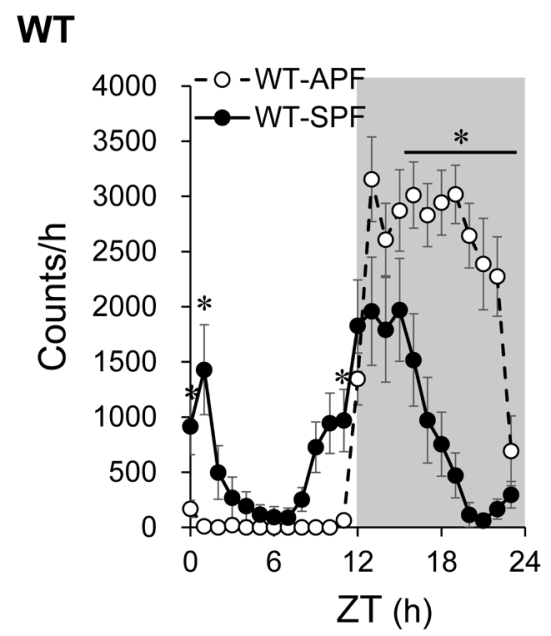

Clk/CIk

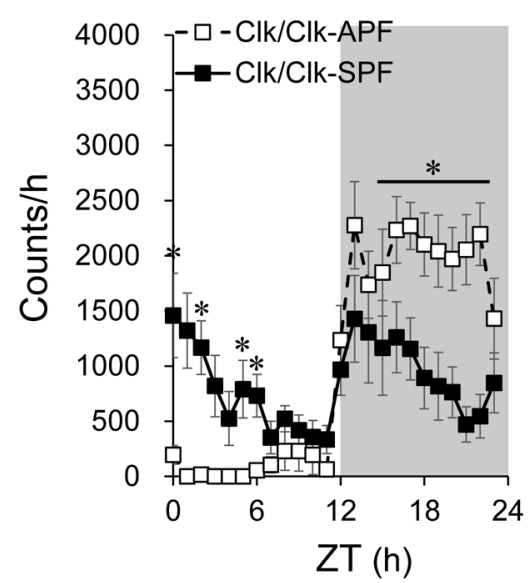

Fig. 1. Circadian Rhythm of Wheel-Running Activity

Hourly values for wheel running by wild-type (WT) and Clock mutant $(\mathrm{Clk} / \mathrm{Clk}$ ) mice the consumed food during nighttime (APF) and daytime (SPF). Values for final three days of restricted food consumption period are averaged. Data are shown as means \pm S.E.M. $(n=9-10) . * p<0.05$; significant differences between APF and SPF mice at each time point. Gray shading, dark period. ZT, Zeitgeber time.

(w/w) fat (54.5\% of total calories), $20.7 \%(w / w)$ carbohydrates (28.3\% of total calories containing $20.0 \%$ (w/w) sucrose). Wild-type and Clock mutant mice were grouped according to whether access to the diet was given only during the sleep phase (SPF; ZT2-10) or only during the active phase (APF; ZT14-22) for $10 \mathrm{~d}$. The mice were weighed on days $0,1,3,6$, and 8 and sacrificed at ZT8 or ZT20 on day 10. Whole blood from mice under inhalational anesthesia was collected into ethylenediaminetetraacetic acid (EDTA)-coated tubes, then dissected liver and epididymal white adipose tissues (WAT) were weighed and rapidly placed in liquid nitrogen. All animal experiments were performed according to the guidelines for animal experiments of the National Institute of Advanced Industrial Science and Technology (AIST). Our institutional Animal Care and Use Committee approved all experimental protocols (Permission \#2017-020).

Measurements of Blood Hormones and Metabolic Parameters Plasma immediately separated from blood samples by centrifugation for $15 \mathrm{~min}$ at $5800 \times \boldsymbol{g}$ was stored at $-80^{\circ} \mathrm{C}$ before concentrations of insulin and leptin were measured and metabolic parameters such as glucose, free fatty acids (FFA), triglycerides (TG), and total cholesterol (T-Cho) were assessed as described. ${ }^{10)}$

Measurements of Liver Lipids Hepatic lipids were assayed as described. ${ }^{10)}$

Real-Time RT-PCR Total RNA was extracted using RNAiso Plus (TaKaRa Bio, Shiga, Japan). Single-stranded cDNA was synthesized using PrimeScript ${ }^{\mathrm{TM}}$ RT reagent kits with gDNA Eraser (TaKaRa Bio). Real-time RT-PCR proceeded using SYBR Premix Ex Taq ${ }^{\mathrm{TM}}$ II (TaKaRa Bio) using the described primers ${ }^{10)}$ and a LightCycler ${ }^{\mathrm{TM}}$ (Roche Diagnostics, Mannheim, Germany). The amount of target mRNA was normalized relative to that of $18 \mathrm{~S}$ ribosomal RNA (rRNA) (Rn18s).

Statistical Analysis All data are expressed as means \pm standard error of the mean (S.E.M.). Data from wheel-running were compared between the APF and SPF groups at various times of the day using Student $t$-tests. Other data were statistically evaluated by 2-way and 3-way ANOVA and Tukey's multiple comparison tests using Excel-Tokei 2010 software (Social Survey Research Information Co., Ltd.,
Osaka, Japan). Values with $p<0.05$ were considered significantly and statistically different.

\section{RESULTS}

Wheel-Running by Wild-Type and Clock Mutant Mice under Time-Restricted Food Consumption Day-night activity rhythms were robust under APF in both wild-type and Clock mutant mice, although the total daily activity was reduced by $18.5 \%$ in the mutants (Fig. 1). Daytime activity in both mouse genotypes was significantly increased under daytime food consumption, whereas a nocturnal increase in activity was maintained in both genotypes. The SPF-induced increase in daytime activity was more evident in mutant, than in wild-type mice. The total daily amounts of time that wildtype and Clock mutant mice spent running on wheels during restricted food consumption were 38.8 and $16.6 \%$ lower for SPF than for APF, respectively.

Daytime Food Consumption Caused Both Wild-Type and Clock Mutant Mice to Become Obese Figure 2 shows that wild-type and Clock mutant mice gained 8.8 and $4.0 \%$ more weight under SPF than APF, respectively. The dominant negative Clock gene mutation obviously attenuated the weight gain caused by SPF (Supplementary Fig. 1A). After $8 \mathrm{~d}$ of restricting food access to either APF or SPF, both mouse genotypes consumed similar amounts of food (Supplementary Fig. 1B), although a transient reduction in food consumption was more obviously caused by SPF in the wild-type mice. Daytime food consumption did not affect relative liver weight in either genotype, but livers were significantly lighter in Clock mutant than wild-type mice under both APF and SPF (Fig. 2C). The relative weight of WAT was respectively 57.9 and $70.2 \%$ heavier under SPF than APF, in wild-type and Clock mutant mice (Fig. 2D).

Daytime Food Consumption Resulted in Hyperinsulinemia, Hyperleptinemia and Hepatic Lipid Accumulation in Wild-Type and Clock Mutant Mice We previously found that SPF induces hyperinsulinemia and hyperleptinemia. ${ }^{10,12)}$ Therefore, we measured plasma insulin and leptin concentrations in wild-type and Clock mutant mice under APF and SPF 

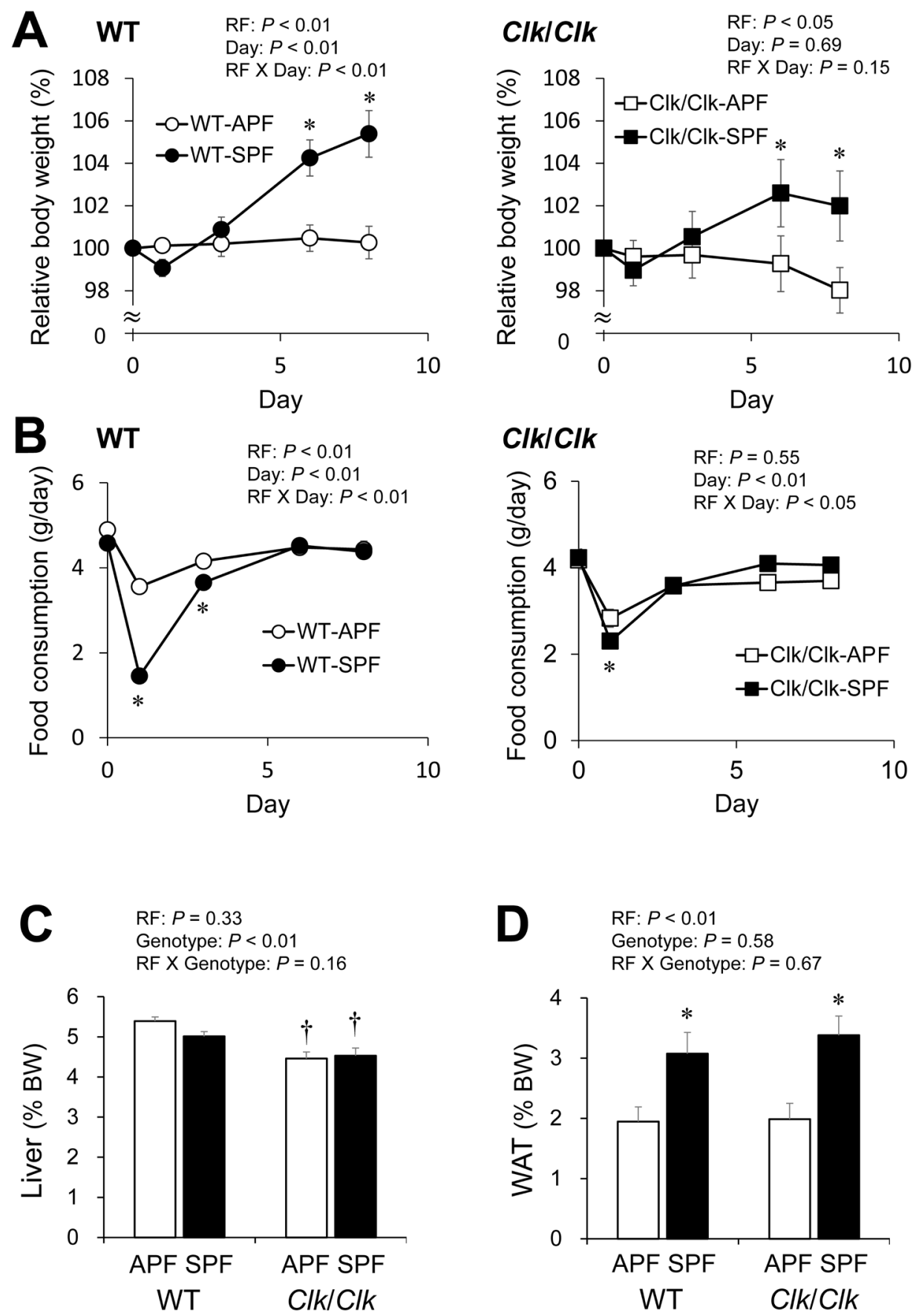

Fig. 2. Body Weight, Food Consumption, and Relative Weight of Liver and Adipose Tissues to Body Weight

Body weight (A) and daily food consumption (B) were measured in wild-type (WT) and Clock mutant (Clk/Clk) mice under food consumption during nighttime active phase (APF) or daytime sleep phase (SPF). Data are shown as means \pm S.E.M. $(n=10-23)$. $* p<0.05$, significant differences between APF and SPF mice on corresponding day. ${ }^{\dagger} p<0.05$, significant differences between Day 0 and Days $1-8$. Relative weight of liver (C) and white adipose tissues (WAT) (D) to body weight at end of restricted food consumption. Data are shown as means \pm S.E.M. $(n=10-23)$. Significant differences between * APF and SPF mice and between ${ }^{\dagger}$ genotypes $($ both $p<0.05)$.

(Figs. 3A, B). Both concentrations were far higher at ZT8 than at ZT20 under SPF, but similar between ZT8 and ZT20 under APF in both genotypes. Plasma insulin and leptin levels were remarkably increased during the period of restricted food consumption under SPF in both genotypes, and there were no significant differences between genotypes (Supplementary Table 1).

Blood metabolic parameters were evaluated under APF and SPF (Supplementary Fig. 2). Plasma TG concentrations significantly fluctuated in accordance with food consumption rhythms under SPF, but not under APF, in both genotypes (Supplementary Table 2). Daytime food consumption seemed to slightly increase plasma glucose and T-Cho concentrations in both genotypes (Supplementary Table 2).

We measured hepatic TG, FFA, and T-Cho accumulation in wild-type and Clock mutant mice under APF and SPF (Figs. $3 \mathrm{C}-\mathrm{E}$ ). Hepatic TG values were significantly higher under SPF than APF, whereas FFA and T-Cho values did not significantly differ between these conditions in both mouse genotypes (Supplementary Table 2).

Daytime Food Consumption Induced Lipogenic Gene Expression in Liver and WAT of Wild-Type and Clock Mutant Mice We evaluated the mRNA expression of genes 

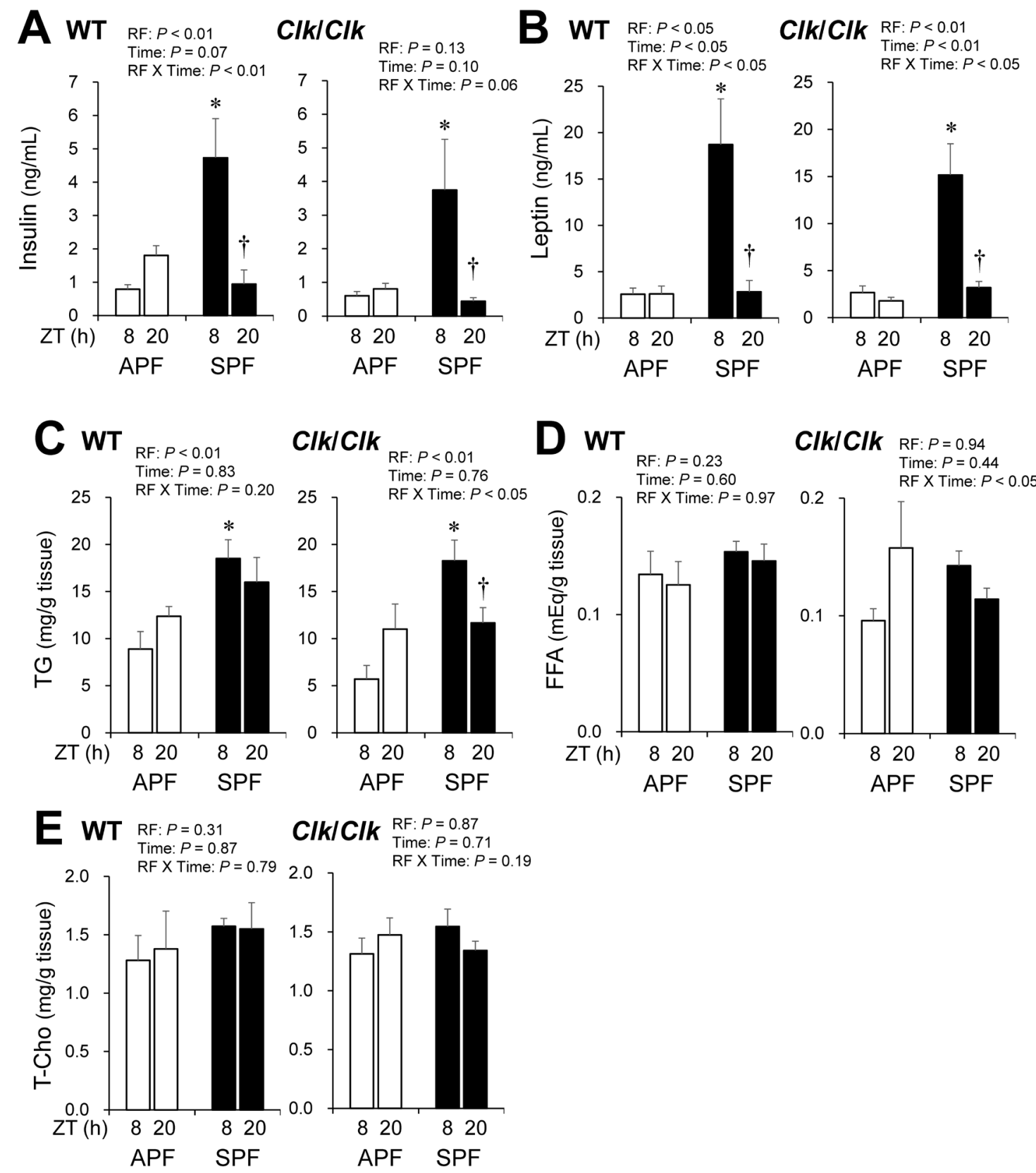

Fig. 3. Concentrations of Plasma Insulin (A) and Leptin (B), and Hepatic Triglycerides (C), Free Fatty Acids (D) and Total Cholesterol (E)

Wild-type (WT) and Clock mutant (Clk/Clk) mice were allowed to consume food during nighttime active phase (APF) or daytime sleep phase (SPF) for 10 d. Concentrations of plasma hormones and hepatic lipids at ZT8 and ZT20. Data are shown as means \pm S.E.M. $(n=4-6)$. Significant differences between $*$ APF and SPF mice and ${ }^{\dagger}$ ZT8 and ZT20 ( $p<0.05$ for both). FFA, free fatty acids; T-Cho, total cholesterol, TG, triglyceride; ZT, Zeitgeber time.

involved in fatty acid synthesis in the liver (Fig. 4) and WAT (Supplementary Fig. 3) of APF and SPF mice. Day-night expression profiles of Fasn and $S c d 1$ mRNA in the liver significantly differed between APF and SPF in both mouse genotypes. The mRNA expression of these genes was higher at ZT8 than at ZT20 under SP, but similar between the 2 time points under AP. Three-way ANOVA and post hoc comparisons revealed significantly elevated mRNA expression of these genes under SPF than APF in both genotypes (Supplementary Table 3). More Acaca and Fasn mRNA tended to be expressed in the WAT of both genotypes under SPF than APF (Supplementary Table 4).

Daytime Food Consumption Gene-Dependently Affected Rhythmic Expression of Circadian Clock Genes in Wild-Type and Clock Mutant Mice We analyzed temporal expression profiles of circadian clock genes Perl and Per2, and the clock-controlled gene $D b p$ in the liver (Supplementary Fig. 4) and WAT (Supplementary Fig. 5). The timing of food consumption significantly affected the day-night expression rhythms of Per2, but not Perl in the liver and WAT of both genotypes. Expression levels of Perl and Per2 in the liver were similar between both genotypes, whereas the Clock mutation obviously reduced those of $\mathrm{Dbp}$ (Supplementary Table 3).

\section{DISCUSSION}

Food consumption at an uncommon time of day might be associated with obesity and metabolic disruption in both experimental animals and humans. We previously found more weight gain and adiposity accompanied by hyperinsulinemia 

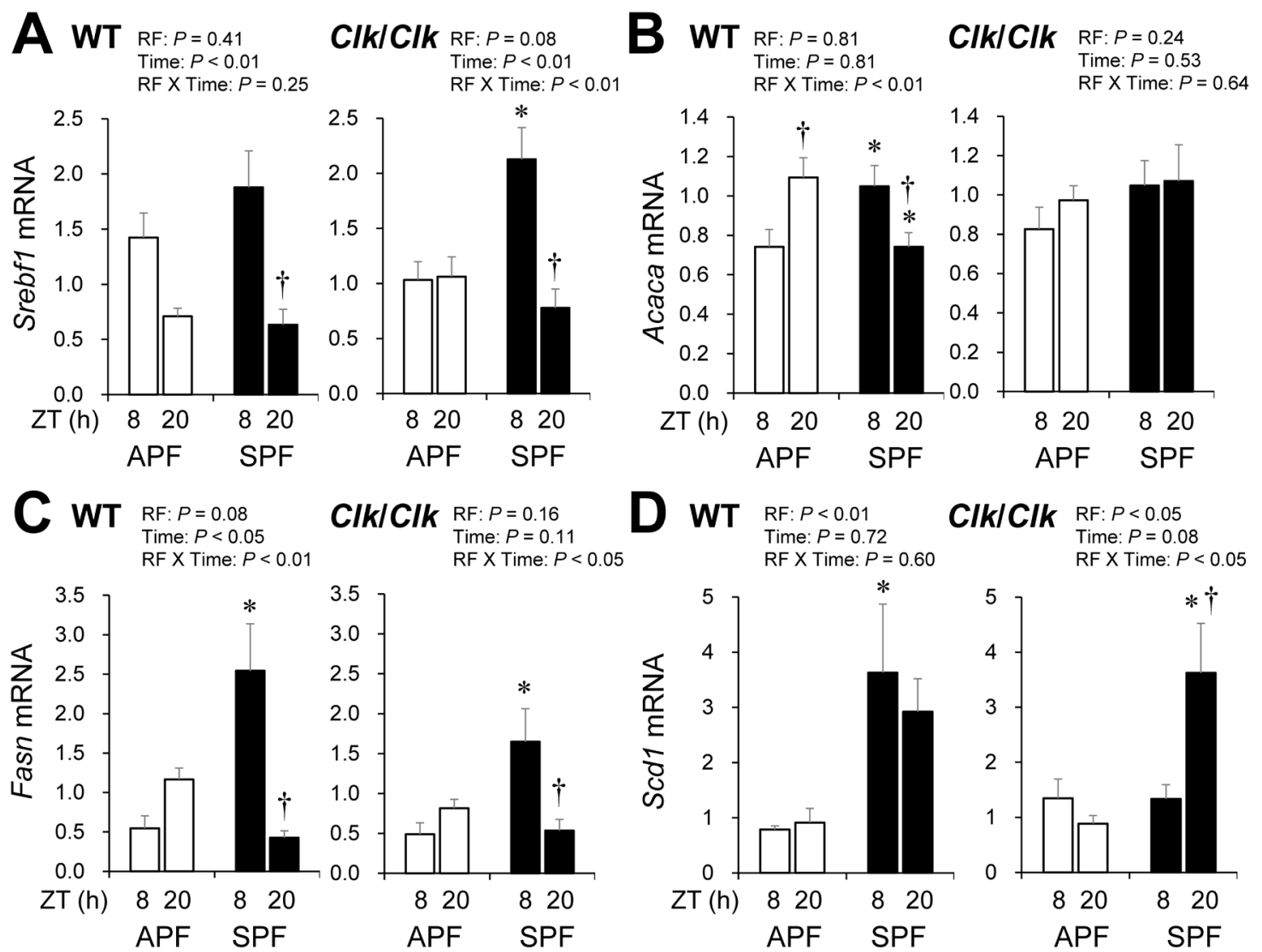

Fig. 4. Temporal Expression Profiles of Genes Associated with Hepatic Lipogenesis

Wild-type (WT) and Clock mutant (Clk/Clk) mice were allowed to consume food during nighttime active phase (APF) or daytime sleep phase (SPF) for 10 d. Temporal mRNA expression was determined at ZT8 and ZT20. Data are shown as means \pm S.E.M. $(n=4-6)$. Significant differences between $*$ APF and SPF mice and ${ }^{\dagger}$ ZT8 and ZT20 ( $p<0.05$ for both). ZT, Zeitgeber time.

and hyperleptinemia in SPF, compared with APF mice. ${ }^{12)}$ We sought to clarify whether the molecular circadian clock is involved in the underlying mechanisms of SPF-induced obesity and metabolic disorders such as hyperinsulinemia, hyperleptinemia and adiposity.

Hundreds of circadian genes that regulate glucose and lipid metabolism have been identified as the transcriptional targets of CLOCK in the mouse liver using DNA microarray technology. ${ }^{15)}$ Such findings suggest that Clock is involved in metabolic regulation as well as being a core component of the circadian clock. Dominant negative Clock gene mutation attenuates body weight gain, intestinal lipid absorption, and hepatic lipid accumulation caused by a high-fat diet in mice on an ICR background, ${ }^{16,17)}$ but these effects are reversed in mice with a C57BL/6J background. ${ }^{18,19)}$ The present study showed that SPF increased body weight, adipose tissue mass, lipogenic gene expression in metabolic tissues, and induced hyperinsulinemia, hyperleptinemia and hepatic lipid accumulation in wild-type and Clock mutant mice with an ICR background. These findings suggest that the molecular clock in nocturnal mice is not essentially associated with the metabolic disruption caused by consuming food during the daytime.

On the other hand, the Clock mutation tended to attenuate gains in body weight after $6 \mathrm{~d}$ under both APF and SPF. Clock mutant mice are sensitive to fasting and starvation because they have impaired hepatic glycogen synthesis. ${ }^{20)}$ The significantly lower ratio of liver weight to body weight in the Clock mutant, compared with wild-type mice under both APF and
SPF (Fig. 2C), might reflect impaired glycogen synthesis.

We recently showed that leptin resistance is involved in the underlying mechanisms of the obesity and metabolic disruption caused by consuming food during SPF. ${ }^{10)}$ The gains in body weight, adipose tissue mass, lipogenic gene expression in metabolic tissues, and hepatic lipid accumulation caused by SPF are abolished in leptin-resistant $d b / d b$ mice. We also found that leptin-deficient $o b / o b$ mice were significantly more sensitive to leptin under SPF than APF, suggesting that the circadian fluctuation of central leptin sensitivity that increases during the resting phase is associated with SPF-triggered leptin resistance, which causes obesity in mice. ${ }^{10)}$ The present study found that SPF seemed to cause leptin resistance in both wild-type and Clock mutant mice, because SPF induced hyperleptinemia in both genotypes without affecting the amount of food that the mice consumed. We also found that CLOCK is not involved in the transcriptional activation of the Lep gene under SPF (Supplementary Fig. 4). These observations suggested that SPF-induced leptin resistance is CLOCK-independent. Homozygous Clock mutant mice have abnormally long free-running rhythmic periods of circadian locomotor activity, but these are not abolished in mice with an ICR background. ${ }^{13)}$ NPAS2 is a paralog of CLOCK that dimerizes with BMAL1 to form transcriptionally active complexes and might compensate for central circadian leptin sensitivity because Npas 2 and Clock double-knockout leads to completely arrhythmic behavior and clock gene expression in the SCN. ${ }^{21}$

The present study showed that the rhythmic expression 
of Per2, but not Perl, mRNA was synchronized to recurrent food consumption in the livers of wild-type and Clock mutant mice (Supplementary Fig. 3), whereas the timing of food consumption did not affect such fluctuations in skeletal muscle as described (data not shown). ${ }^{10-12)}$ CLOCK transcriptionally activates Perl and Per2 genes as well as Dbp. ${ }^{3)}$ Therefore, the mRNA expression of these genes was damped at least under food consumption ad libitum. ${ }^{22}$ Here, the rhythmic expression of Per2 mRNA was similarly affected by the feeding time in both wild-type and Clock mutant mice, suggesting that CLOCK-independent food-entrainable peripheral circadian oscillators were evoked under both APF and SPF. ${ }^{13,23)}$ Timerestricted food consumption differentially affects peripheral clocks in the liver and skeletal muscle, suggesting that peripheral metabolic rhythms are not coupled between these tissues. ${ }^{12)}$ Desynchronized metabolic rhythms in peripheral tissues might be responsible for the metabolic disruption in SPF mice.

The present study showed that the molecular clock is not essentially involved in the metabolic disruption caused by SPF in mice. It should be noted that Clock mutation affect the phase of various circadian rhythms, such as body temperature, hormonal secretion, blood metabolic parameters, and metabolic gene expressions. ${ }^{14,15,24)}$ one of the limitations of the present study was that we observed the metabolic phenotypes at only two time points. Therefore, the molecular clock might partly affect the metabolic disruptions caused by SPF. Desynchronization between the timing of food consumption and central clock-dependent behaviour as well as metabolic rhythms might be responsible for the metabolic disruption caused by SPF in mice.

Acknowledgments This study was supported by the Japan Society for the Promotion of Science (JSPS) KAKENHI Grant Number JP16K00940 (to K. Oishi).

Conflict of Interest The authors declare no conflict of interest.

Supplementary Materials The online version of this article contains supplementary materials.

\section{REFERENCES}

1) Dibner C, Schibler U, Albrecht U. The mammalian circadian timing system: organization and coordination of central and peripheral clocks. Annu. Rev. Physiol., 72, 517-549 (2010).

2) Maury E, Ramsey KM, Bass J. Circadian rhythms and metabolic syndrome: from experimental genetics to human disease. Circ. Res., 106, 447-462 (2010).

3) Buhr ED, Takahashi JS. Molecular components of the mammalian circadian clock. Handbook Exp. Pharmacol., 217, 3-27 (2013).

4) Vitaterna MH, King DP, Chang AM, Kornhauser JM, Lowrey PL, McDonald JD, Dove WF, Pinto LH, Turek FW, Takahashi JS. Mutagenesis and mapping of a mouse gene, Clock, essential for circadian behavior. Science, 264, 719-725 (1994).

5) King DP, Takahashi JS. Forward genetic approaches to circadian clocks in mice. Cold Spring Harb. Symp. Quant. Biol., 61, 295-302 (1996).

6) Schibler U, Ripperger J, Brown SA. Peripheral circadian oscillators in mammals: time and food. J. Biol. Rhythms, 18, 250-260 (2003).

7) Shibata S, Tahara Y, Hirao A. The adjustment and manipulation of biological rhythms by light, nutrition, and abused drugs. Adv. Drug Deliv. Rev., 62, 918-927 (2010).

8) Garaulet M, Gomez-Abellan P. Timing of food intake and obesity: a novel association. Physiol. Behav., 134, 44-50 (2014).

9) Moran-Ramos S, Baez-Ruiz A, Buijs RM, Escobar C. When to eat? The influence of circadian rhythms on metabolic health: are animal studies providing the evidence? Nutr. Res. Rev., 29, 180-193 (2016).

10) Oishi K, Hashimoto C. Short-term time-restricted feeding during the resting phase is sufficient to induce leptin resistance that contributes to development of obesity and metabolic disorders in mice. Chronobiol. Int., 35, 1576-1594 (2018).

11) Okauchi H, Hashimoto C, Nakao R, Oishi K. Timing of food intake is more potent than habitual voluntary exercise to prevent dietinduced obesity in mice. Chronobiol. Int., 36, 57-74 (2019).

12) Yasumoto $Y$, Hashimoto $C$, Nakao R, Yamazaki $H$, Hiroyama $H$, Nemoto T, Yamamoto S, Sakurai M, Oike H, Wada N, YoshidaNoro C, Oishi K. Short-term feeding at the wrong time is sufficient to desynchronize peripheral clocks and induce obesity with hyperphagia, physical inactivity and metabolic disorders in mice. Metabolism, 65, 714-727 (2016).

13) Oishi K, Miyazaki K, Ishida N. Functional CLOCK is not involved in the entrainment of peripheral clocks to the restricted feeding: entrainable expression of mPer2 and BMAL1 mRNAs in the heart of Clock mutant mice on Jcl:ICR background. Biochem. Biophys. Res. Commun., 298, 198-202 (2002).

14) Sei H, Oishi K, Morita Y, Ishida N. Mouse model for morningness/ eveningness. Neuroreport, 12, 1461-1464 (2001).

15) Oishi K, Miyazaki K, Kadota K, Kikuno R, Nagase T, Atsumi G, Ohkura N, Azama T, Mesaki M, Yukimasa S, Kobayashi H, Iitaka C, Umehara T, Horikoshi M, Kudo T, Shimizu Y, Yano M, Monden M, Machida K, Matsuda J, Horie S, Todo T, Ishida N. Genome-wide expression analysis of mouse liver reveals CLOCK-regulated circadian output genes. J. Biol. Chem., 278, 41519-41527 (2003).

16) Kudo T, Tamagawa T, Kawashima M, Mito N, Shibata S. Attenuating effect of clock mutation on triglyceride contents in the ICR mouse liver under a high-fat diet. J. Biol. Rhythms, 22, 312-323 (2007).

17) Oishi K, Atsumi G, Sugiyama S, Kodomari I, Kasamatsu M, Machida K, Ishida N. Disrupted fat absorption attenuates obesity induced by a high-fat diet in Clock mutant mice. FEBS Lett., 580, 127-130 (2006).

18) Oishi K, Ohkura N, Wakabayashi M, Shirai H, Sato K, Matsuda J, Atsumi G, Ishida N. CLOCK is involved in obesity-induced disordered fibrinolysis in $o b / o b$ mice by regulating $P A I-1$ gene expression. J. Thromb. Haemost., 4, 1774-1780 (2006).

19) Turek FW, Joshu C, Kohsaka A, Lin E, Ivanova G, McDearmon E, Laposky A, Losee-Olson S, Easton A, Jensen DR, Eckel RH, Takahashi JS, Bass J. Obesity and metabolic syndrome in circadian Clock mutant mice. Science, 308, 1043-1045 (2005).

20) Doi R, Oishi K, Ishida N. CLOCK regulates circadian rhythms of hepatic glycogen synthesis through transcriptional activation of Gys2. J. Biol. Chem., 285, 22114-22121 (2010).

21) DeBruyne JP, Weaver DR, Reppert SM. CLOCK and NPAS2 have overlapping roles in the suprachiasmatic circadian clock. Nat. Neurosci., 10, 543-545 (2007).

22) Oishi K, Fukui H, Ishida N. Rhythmic expression of BMAL1 mRNA is altered in Clock mutant mice: differential regulation in the suprachiasmatic nucleus and peripheral tissues. Biochem. Biophys. Res. Commun., 268, 164-171 (2000).

23) Mukherji A, Kobiita A, Chambon P. Shifting the feeding of mice to the rest phase creates metabolic alterations, which, on their own, shift the peripheral circadian clocks by 12 hours. Proc. Natl. Acad. Sci. U.S.A., 112, E6683-E6690 (2015).

24) Oishi K, Ohkura N, Kadota K, Kasamatsu M, Shibusawa K, Matsuda J, Machida K, Horie S, Ishida N. Clock mutation affects circadian regulation of circulating blood cells. J. Circadian Rhythms, 4, 13 (2006). 